\title{
基于微波亮温及集合Kalman滤波的土壤湿度 同化方案
}

\author{
(1) 中国科学院大气物理研究所, 北京 100029 ; \\ (2) 中国科学院研究生院, 北京 100049 ; \\ (3) 中国气象局国家卫星气象中心, 北京 100081 \\ * 联系人, E-mail: zxie@lasg.iap.ac.cn
}

贾炳浩 ${ }^{(1)}$, 谢正辉 ${ }^{(1)}$, 田向军 ${ }^{(1)}$, 师春香 ${ }^{(3)}$

收稿日期: 2009-01-16; 接受日期: 2009-09-24

国家重点基础研究发展计划项目(编号: 2010CB428403, 2009CB421407)、财政部/科技部公益类行业专项(编号: GYHY200706005)和国家高 技术研究发展计划项目(编号: 2007AA12Z144, 2009AA12Z129)资助

\begin{abstract}
摘要 基于集合 Kalman 滤波及 SCE-UA(shuffled complex evolution)算法发展了能够直 接同化微波亮温的土壤湿度同化方案。该方案以陆面过程模式 CLM 3.0 中的土壤水模型 作为预报算子，以辐射传输模型作为观测算子。整个同化过程分为参数优化和土壤湿度 同化两个阶段, 利用 SCE-UA 算法优化辐射传输模型中难以确定的植被光学厚度参数和 地表粗䊁度参数, 并利用优化参数作为观测算子的模型参数进行同化. 通过人工理想试 验表明该同化方案可以明显改善表层土壤湿度的模拟精度，并且对深层土壤湿度的模拟 也有一定程度的改善; 利用 AMSR-E 亮温(10.65 GHz 垂直极化)所进行的实际同化试验表 明顶层 $(0 \sim 10 \mathrm{~cm})$ 土壤湿度同化结果与观测的均方根误差 $(R M S E)$ 由模拟的 0.05052 减小到 0.03355 , 相对减小了 $33.6 \%$, 而较深层 $(10 \sim 50 \mathrm{~cm})$ 平均减小了 $20.9 \%$. 这些同化试验显示 该同化方案的合理性.
\end{abstract}

关键词

陆面数据同化

土壤湿度

集合 Kalman 滤波

SCE-UA 算法

辐射传输模型

AMSR-E
土壤湿度在陆气相互作用中起着重要的作用, 它通过改变地表能量中感热和潜热通量的分配 ${ }^{[1,2]}$, 从而对天气、气候产生较大影响 ${ }^{[3]}$. 因此, 具有一定 精度和时空分布的土壤湿度资料对于天气与气候模 式的发展意义重大，而目前观测的土壤湿度相当稀 少, 且均为不连续分布, 时间间隔长, 其适用性受到 很大的限制. 另一方面, 由于受各种物理过程参数化 方案以及大气强迫等不确定因素的限制, 利用陆面 过程模型模拟的土壤湿度还难以达到满意的精度 ${ }^{[4]}$.
微波遥感(特别是低频)由于对表层土壤湿度信息比 较敏感 ${ }^{[5]}$, 可以通过各种反演方法来估计几到十几厘 米土壤湿度的分布 ${ }^{[6 \sim 8]}$, 但它对于更深层土壤湿度却 无能为力，且其精度也存在一定的问题.

陆面数据同化起源于大气和海洋同化, 它能够 充分利用陆面模式和各种观测信息(如站点观测、遥 感资料等)得到陆面变量的最优估计. 近年来, 人们 通过同化土壤湿度站点观测资料来改善土壤湿度估 计 $^{[9 \sim 14]}$. De Lannoy 等 ${ }^{[15]}$ 基于陆面过程模型 CLM 2.0

引用格式: Jia B H, Xie Z H, Tian X J, et al. A soil moisture assimilation scheme based on the ensemble Kalman filter using microwave brightness temperature. Sci China Ser D-Earth Sci, 2009, 52: 1835-1848, doi: 10.1007/s11430-009-0122-Z 
和集合 Kalman 滤波方法分析了同化深度和同化频率 对土壤湿度同化结果的影响; 为了考虑土壤冻融过 程和次网格变异性, Tian 等 ${ }^{[16,17]}$ 分别采用 DualEnsemble Kalman 滤波和 Dual-Unscent Kalman 滤波 算法构建同时优化模型参数和状态变量的双通优化 框架进行同化试验, 结果表明它不仅可以提高表层 土壤湿度模拟精度, 并且对深层土壤湿度也有一定 程度地改善.

具有较高时间、空间分辨率的 SSM/I, TMI, AMSR-E 等微波遥感数据极易获得, 其在陆面数据同 化系统中的应用也逐渐成为研究的热点. Crosson 等 ${ }^{[18]}$ 结合分布式水文模型 SHEELS 和扩展 Kalman 滤 波算法, 利用微波辐射传输模型同化 $\mathrm{S}$ 和 $\mathrm{L}$ 波段微波 亮温, 在降雨数据不准确的情况下, 同化后土壤湿度 廓线比模式模拟更接近观测. $\mathrm{Li}$ 等 ${ }^{[19]}$ 提出了用极快速 模拟再退火算法和 SiB2 模型同化 TMI 低频亮温(10.7 $\mathrm{GHz})$, Huang 等 ${ }^{[20]}$ 在此基础上用集合 Kalman 滤波同 化算法针对青藏高原也进行了类似试验. 这些方案 均表明同化微波亮温可以改善模式模拟的土壤湿度 分布. 然而研究表明 ${ }^{[21 ~ 24]}$ : 植被和地表非均匀性对 辐射传输模型模拟的亮温有很大影响, 并且它们具 有较大的不确定性, 因而获得一组合适的植被光学 厚度和地表粗粘度参数对陆面数据同化中微波遥感 数据的应用具有重要意义. 为此, Yang 等 ${ }^{[25]}$ 发展了 同化 AMSR-E 垂直极化 6.9 和 $18.7 \mathrm{GHz}$ 亮温估计土 壤水分和能量平衡的自动率定系统.

在 Yang 等 ${ }^{[25]}$ 工作的基础上, 本文发展了一个基 于集合 Kalman 滤波方法 ${ }^{[26]}$ 和 SCE-UA(shuffled complex evolution)优化算法 ${ }^{[27]}$ 的双通土壤湿度同化 方案. 它利用土壤水模型作为预报算子估计土壤湿 度的分布, 并利用辐射传输模型作为观测算子同化 微波亮温, 从而实现与陆面过程模型 CLM 3. $0^{[28]}$ 的 “离线”耦合. 整个同化方案包括参数优化和土壤湿 度同化两个阶段, 本文与 Yang 等 ${ }^{[25]}$ 都采用双通优化 的框架, 但本研究的优化系统是嵌入在集合 Kalman 滤波这一较为先进且在陆面数据同化中被广泛应用 的同化方法; 另外, 此同化方案在参数优化阶段并未 使用土壤湿度指数(soil wetness index, SWI) ${ }^{[25]}$ 调整驱 动辐射传输模型的表层土壤湿度, 而是对参数优化 也采用了随机给定参数初始值以消除初始值的设置 对最终的优化结果影响. 本文将通过一个人工理想 试验来验证其可行性, 并利用 AMSR-E 亮温资料进
行实际同化试验.

\section{1 模型和方法}

本研究发展的双通土壤湿度同化方案采用美国 国家大气研究中心 NCAR 的陆面过程模型 Community Land Model Version 3.0(简写为 CLM 3.0) [ $^{[28]}$ 作 为平台，它包括预报算子、观测算子、算法(同化和优 化)和观测数据等, 一维土壤水模型作为预报算子模 拟土壤湿度的分布, 微波辐射传输模型作为观测算 子模拟微波亮温，同化算法采用目前较为流行的平 方根分析集合 Kalman 滤波方法 ${ }^{[26]}$, SCE-UA 算法 ${ }^{[27]}$ 被用来优化辐射传输模型中植被光学厚度和地表粗 糙度参数, 输入数据包括大气强迫数据和微波亮温 (本文取 $10.65 \mathrm{GHz}$ )以及所需的地表参数集(土壤质 地、叶面积指数等). 由于垂直极化相对于水平极化对 植被不太敏感 ${ }^{[29]}$, 故本研究选取垂直极化亮温.

\section{1 土壤水模型}

为了计算方便 (不考虑水热耦合和冻融过程), 模 型算子采用 CLM 3.0 中使用的一维土壤水垂直运动 方程 ${ }^{[28]}$ (忽略水平流动):

$$
\frac{\partial \theta}{\partial t}=-\frac{\partial q}{\partial z}-E-R_{\mathrm{fm}},
$$

其中, $\theta$ 是土壤体积含水量 $\left(\mathrm{m}^{3} \cdot \mathrm{m}^{-3}\right), q$ 是垂直土壤水 通量 $\left(\mathrm{mm} \cdot \mathrm{s}^{-1}\right), E$ 是蒸发速率 $\left(\mathrm{mm} \cdot \mathrm{s}^{-1}\right), R_{\mathrm{fm}}$ 是融化 (负 值)或冻结(正值)速率(为简便, 本研究中 $R_{\mathrm{fm}}$ 取为零), $z$ 是到地面的垂直距离 ( $q$ 和 $z$ 取向下为正). 土壤水分 通量 $q$ 可根据 Darcy 定律 ${ }^{[30]}$ 来计算:

$$
q=-k \frac{\partial(\psi+z)}{\partial z},
$$

这里, $k=k_{\mathrm{s}}\left(\theta / \theta_{\mathrm{s}}\right)^{2 b+3}$ 是水力传导率, $\psi=\psi_{\mathrm{s}}\left(\theta / \theta_{\mathrm{s}}\right)^{-b}$ 是土 壤水势, $k_{\mathrm{s}}, \psi_{\mathrm{s}}, \theta_{\mathrm{s}}$ 和 $b$ 均为常数. 本文中采用与 CLM 3.0 一样的土壤分层, 即分成 10 层厚度不均的土壤层 (具体见 Oleson 等 ${ }^{[28]}$ ), 采用有限元质量集中法计算土 壤水模型 ${ }^{[31,32]}$. 上边界条件为

$$
q_{0}(t)=-\left.k \frac{\partial(\psi+z)}{\partial z}\right|_{0},
$$

这里, $q_{0}(t)$ 是地表水分通量(文中作为入渗, $E$ 取为零), 下边界通量是零通量, 即 $q_{l}=0$.

\section{2 微波辐射传输模型}

微波辐射传输模型用来模拟大气层顶微波亮温, 
在低频波段(如<20 GHz), 微波信号受大气影响很小, 故本文不考虑大气的削弱作用, 认为植被层顶亮温 约等于大气层顶亮温. 同 Mo 等 ${ }^{[33]}$ 和 Yang 等 ${ }^{[25]}$, 在 没有雪盖的地方, 忽略地表植被间多次散射, 植被层 顶微波亮温根据以下公式给出:

$$
\begin{aligned}
T_{\mathrm{b}, p}= & T_{\mathrm{g}}\left(1-r_{\mathrm{s}, p}\right) \exp \left(-\tau_{\mathrm{c}}\right)+T_{\mathrm{c}}(1-\omega)\left[1-\exp \left(-\tau_{\mathrm{c}}\right)\right] \\
& \cdot\left[1+r_{\mathrm{s}, p} \exp \left(-\tau_{\mathrm{c}}\right)\right],
\end{aligned}
$$

其中, 下标 $p$ 表示极化方向(垂直或水平极化), $r_{\mathrm{s}, p}$ 是 土壤反射率, $\tau_{\mathrm{c}}$ 和 $\omega$ 分别是植被光学厚度和单次散射 反照率, $T_{\mathrm{g}}$ 和 $T_{\mathrm{c}}$ 分别是地表温度和植被温度. 文中将 采用与 Yang 等 ${ }^{[25]}$ 相同的辐射传输模型.

为了考虑地表粗䊁度对微波辐射的影响, 采用 $Q-H$ 模型计算土壤的反射率 ${ }^{[34]}$ :

$$
\begin{aligned}
& r_{s h}=\left[(1-Q) \cdot R_{\mathrm{h}}+Q \cdot R_{\mathrm{v}}\right] \exp (-H), \\
& r_{s v}=\left[(1-Q) \cdot R_{\mathrm{v}}+Q \cdot R_{\mathrm{h}}\right] \exp (-H),
\end{aligned}
$$

其中, $\mathrm{h}$ 和 $\mathrm{v}$ 分别表示垂直和水平极化, $Q$ 和 $H$ 是表示 地表粗鉌度的经验参数, 光滑地表的 Fresnel 反射率 $R_{\mathrm{h}}$ 及 $R_{\mathrm{v}}$ 可用以下公式计算:

$$
\begin{aligned}
& R_{\mathrm{h}}=\left|\frac{\cos \theta-\sqrt{\varepsilon_{r}-\sin ^{2} \theta}}{\cos \theta+\sqrt{\varepsilon_{r}-\sin ^{2} \theta}}\right|^{2}, \\
& R_{\mathrm{v}}=\left|\frac{\varepsilon_{r} \cos \theta-\sqrt{\varepsilon_{r}-\sin ^{2} \theta}}{\varepsilon_{r} \cos \theta+\sqrt{\varepsilon_{r}-\sin ^{2} \theta}}\right|^{2},
\end{aligned}
$$

土壤介电常数 $\varepsilon_{r}$ 的计算见 Dobson 等 ${ }^{[35]}$ 和 Ulaby 等 ${ }^{[36]}$. 在公式(4)和(5)中的植被光学厚度及地表粗糙度等参 数根据以下公式计算 ${ }^{[21,22,29,37]}$ :

$$
\begin{gathered}
Q=Q_{0}(k \cdot \sigma)^{0.795}, \\
H=(k \cdot \sigma)^{\sqrt{0.1 \cos \theta},} \\
\tau_{\mathrm{c}}=b^{\prime}(100 \lambda)^{\chi} w_{\mathrm{c}} / \cos \theta, \\
w_{\mathrm{c}}=\exp (\mathrm{LAI} / 3.3)-1, \\
\varpi=0.00083 / \lambda,
\end{gathered}
$$

其中, $k=2 \pi / \lambda$ 表示波数, $\lambda$ 是波长 $(\mathrm{m}), \sigma$ 表示地表均方 根高度, $\theta$ 是入射角, $w_{\mathrm{c}}$ 是植被含水量 $\left(\mathrm{kg} \cdot \mathrm{m}^{-2}\right), \mathrm{LAI}$ 是 叶面积指数 $\left(\mathrm{m}^{2} \cdot \mathrm{m}^{-2}\right)$, 而 $b^{\prime}, \chi$ 和 $Q_{0}$ 是经验系数.

根据 Jakson 和 Schmugge 等 ${ }^{[21]}, \chi$ 可取为 -1.08 (茎 为主, 如小麦)或者 -1.38 (叶为主, 如大豆). 由于陆面 过程模型 CLM 3.0 中土壤孔隙度可以利用砂土含量 计算, 即 $\theta_{\mathrm{sat}, i}=0.489-0.00126 \cdot(\mathrm{sand} \%)_{i}$ (具体见文献 Oleson 等 ${ }^{[28]}$ 中的公式 7.72), 其中 $\theta_{\mathrm{sat}, i}$ 和 (sand\%) 分别
表示第 $i$ 层的土壤孔隙度和砂土含量. 为避免引入过 多待估计的参数带来较多的不确定性以及计算量, 因此本文的参数优化仅仅针对辐射传输模型中几乎 无法给出参数值的那些参数, 而不把陆面模式里面 的参数也同时优化进来. 由于 $b^{\prime}, \sigma$ 和 $Q_{0}$ 这 3 个参数 在实际中很难确定, 而且它们对模拟的亮温影响很 大, 故本文把它们作为待优化的参数.

\section{3 平方根分析集合Kalman滤波算法}

Evensen $^{[26]}$ 在标准集合 Kalman 滤波 ${ }^{[38]}$ 的基础上 提出了一种新的平方根分析方案, 它在计算分析场 集合时不需要观测扰动, 从而能够减小或消除观测 扰动带来的样本误差, 另外, 该算法在计算分析场的 结构时不需要引入额外的附加假设和近似, 例如不 需要假设观测扰动与状态变量集合扰动的无关性, 也不需要对观测误差协方差矩阵求逆等, 并且该算 法计算也很简便.

从传统 Kalman 滤波分析方程的协方差矩阵更新 出发, 采用平方根算法计算集合状态变量扰动的更 新:

$$
P^{a}=P^{f}-P^{f} H^{\mathrm{T}}\left(H P^{f} H^{\mathrm{T}}+R\right)^{-1} H P^{f},
$$

其中, $H$ 是观测算子, $P$ 和 $R$ 分别是状态误差协方差矩 阵和观测误差协方差矩阵, 上标 $a, f$ 和 $\mathrm{T}$ 分别表示分 析场、预报场和矩阵转置. 假设 $A$ 和 $A^{\prime}$ 分别是集合状 态矩阵和集合扰动矩阵, 本文中状态变量为土壤体 积含水量, 则 $A=\left(\theta_{1}, \theta_{2}, \cdots, \theta_{\mathrm{N}}\right) \in \mathrm{R}^{n \times N}$. 除此之外, 观测向量 $d \in R^{m}$ (本文观测取为微波亮温), $E$ 是观测 扰动集合, 定义矩阵 $S=H A^{\prime}$ 和 $C=S S^{\mathrm{T}}+(N-1) R$. 该 算法按照下面的步骤执行:

(1) 计算出矩阵 $C$, 然后对 $C$ 进行特征值分解, 即 $Z \Lambda Z^{\mathrm{T}}=C$;

(2) 更新状态变量的集合平均值 $\overline{\theta^{a}}=$ $\overline{\theta^{f}}+A^{\prime} S^{\mathrm{T}} Z \Lambda^{-1} Z^{\mathrm{T}}\left(d-H \overline{\theta^{f}}\right)$;

(3) 计算矩阵 $X_{2}=\Lambda^{-1 / 2} Z^{\mathrm{T}} S$;

(4) 进行 SVD 分解 $U_{2} \Sigma_{2} V_{2}^{\mathrm{T}}=X_{2}$;

(5) 求解状态变量的分析集合扰动 $A^{a^{\prime}}=A^{\prime} V_{2} \sqrt{I-\Sigma_{2}^{\mathrm{T}} \Sigma_{2}} \Theta(\Theta$ 是任意正交矩阵 $)$, 然后再 加上(2)计算得到的集合分析平均值 $\overline{\theta^{a}}$, 便可得到状 态变量的分析场 $A^{a}$. 


\subsection{SCE-UA优化算法}

SCE-UA 算法是 Duan 等 ${ }^{[27]}$ 在求解概念性降雨径 流模型参数自动率定优化问题时, 针对问题的非线 性、多极值、没有具体的函数表达式和区间型约束等 特点, 提出的一种优化算法. 该算法结合了现有优化 算法(包括遗传算法等)中的一些优点, 可以解决高维 参数的全局优化问题, 且不需要显式目标函数或目 标函数的偏导数等, 它是一种解决非线性约束最优 化问题的有效方法, 可以找到全局最优解. SCE-UA 算法的具体过程及应用详见 Duan 等 ${ }^{[27,39]}$.

\section{5 同化方案流程}

本文中土壤湿度同化方案的流程如图 1 所示, 它 主要分为参数优化和土壤湿度同化两个阶段. 首先 利用大气强迫数据驱动陆面过程模型, 把得到的入 渗作为土壤水模型的上边界条件, 并且把输出的植 被温度、地表温度等作为辐射传输模型的输入, 而辐 射传输模型所需要的土壤质地(砂土和黏土含量)、叶 面积指数等参数来自于陆面过程模型的地表参数集.
（1）参数优化. 本文采用 SCE-UA 优化算法对 1.2 中所描述的辐射传输模型的 3 个模型参数 $b^{\prime}, \sigma$ 和 $Q_{0}$ 进行优化. 定义如下的代价函数:

$$
F=\sum_{t_{1}=1}^{m}\left(T_{b, e s t}^{10.6 V}-T_{b, o b s}^{10.6 V}\right)^{2}
$$

其中, $T_{b, e s t}^{10.6 \mathrm{~V}}$ 和 $T_{b, o b s}^{10.6 \mathrm{~V}}$ 分别是 $10.65 \mathrm{GHz}$ 垂直极化的模 拟和观测亮温. 在一个较长的时间段, 根据观测数分 成 $t_{\text {pass }}$ 个“窗口”(如观测 1 天 1 次, 则 “窗口”长度 $m$ 取 为 $1 \mathrm{~d}$ ), 待优化参数任取一组随机初始值, 有观测时 利用 SCE-UA 优化算法, 通过求解代价函数的最小值 进行参数优化, 可得到 $t_{\text {pass }}$ 组参数值, 最后求出优化 参数在所有窗口的平均值, 即

$$
\begin{gathered}
\overline{b^{\prime}}=\left(1 / t_{\text {pass } 1}\right) \sum_{t_{1}=1}^{t_{\text {pass } 1}} b_{t_{1}}^{\prime}, \quad \bar{\sigma}=\left(1 / t_{\text {pass } 1}\right) \sum_{t_{1}=1}^{t_{\text {pass } 1}} \sigma_{t_{1}}, \\
\overline{Q_{0}}=\left(1 / t_{\text {pass } 1}\right) \sum_{t_{1}=1}^{t_{\text {pass } 1}} Q_{0 t_{1}},
\end{gathered}
$$

作为下一阶段中观测算子的模型参数.

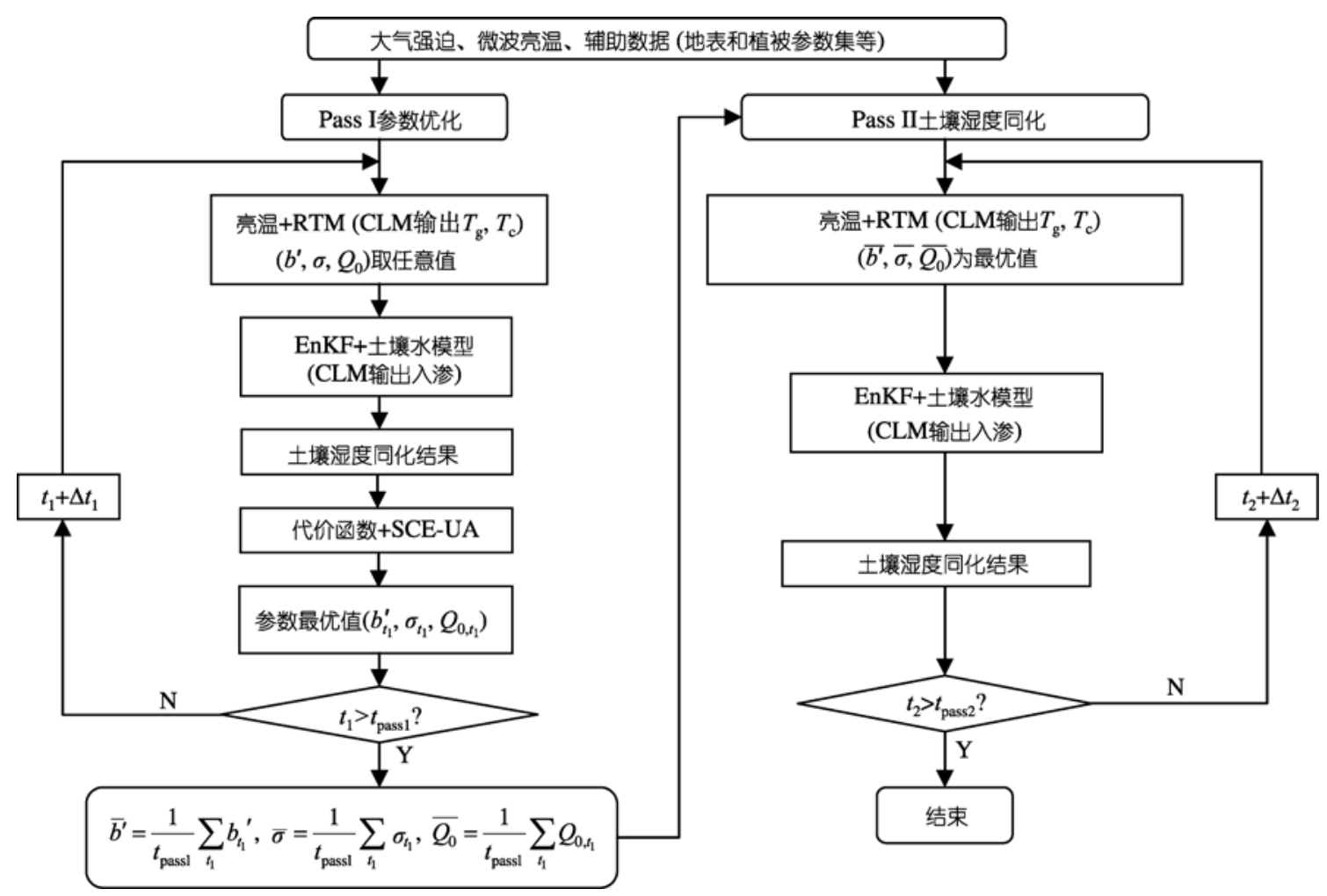

图 1 土壤湿度同化方案流程图

CLM 为陆面过程模型(Community Land Model), RTM 表示辐射传输模型, EnKF 表示集合 Kalman 滤波, $T_{\mathrm{g}}$ 和 $T_{\mathrm{c}}$ 分别是地表温度和植被温度 


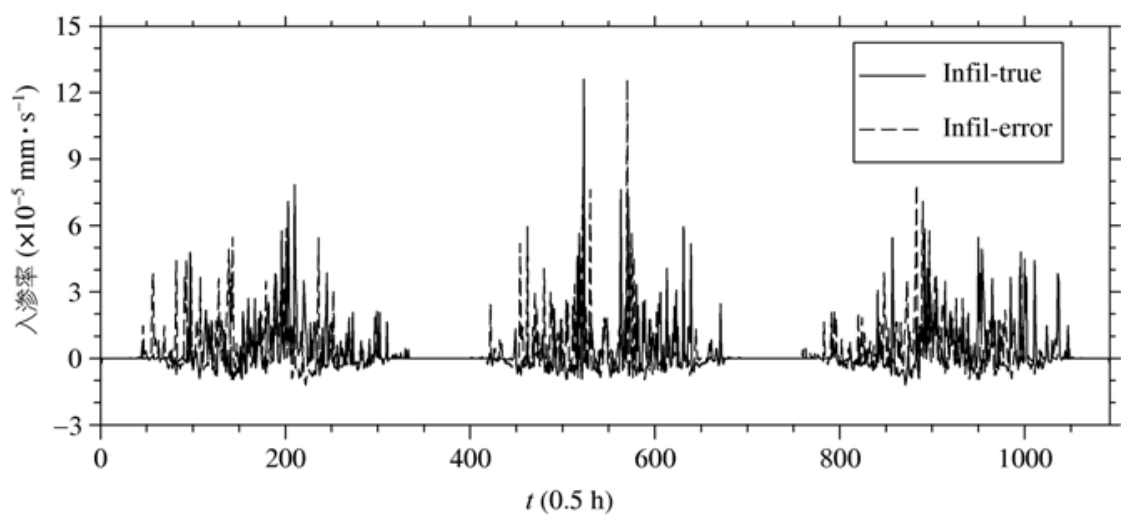

图 2 用于土壤水模型的入渗时间序列

实线表示正确的入渗(Infil-true), 虚线表示有误差的入渗(Infil-error), 横坐标为时间(步长为 $0.5 \mathrm{~h}$ )

(2) 土壤湿度同化. 利用上一阶段优化参数后的 辐射传输模型作为观测算子，在给定时间内基于集 合 Kalman 滤波算法直接同化微波亮温来改善土壤水 模型预报结果，最后得到土壤湿度分布的最优估计.

\section{2 同化试验}

\section{1 理想试验}

为了验证土壤湿度同化方案的可行性, 下面 首先利用 “人造数据”进行理想试验. 选取站点 $\left(110.85^{\circ} \mathrm{E}, 40.85^{\circ} \mathrm{N}\right)$, 并利用基于观测的 $3 \mathrm{~h}$ 大气 强迫数据 ${ }^{[40,41}$ 驱动陆面过程模型 CLM 3.0. 其时间 步长取为 $0.5 \mathrm{~h}$, 在“Spin-up” 10 年之后得到 2002 年 期间共计 1092 时间步的入渗作为真实的输入场 (Infil-true), 并同时获得初始的土壤湿度廓线作为 真实的初始场(Ini-true). 除此之外, 由 CLM3.0 所 生成的植被温度、地表温度等陆面变量以及土壤质 地和叶面积指数等地表参数集作为辅助数据集提 供给辐射传输模型, 计算微波亮温. 在 Ini-true 和 Infil-true 上分别加上随机偏差生成有误差的初始 场(记作 Ini-error)和入渗(记作 Infil-error), 具体见 图 2 和 3. 这里用 Infil-error 表示预报模型有误差.

首先, 在 Ini-true 和 Infil-true 条件下驱动土壤水 模型得到土壤湿度分布作为真实值 (初始场和模式预 报均没有误差), 在 Ini-error 和 Infil-true 下得到土壤 湿度分布作为观测值(初始场有误差，模式预报没有 误差), 在 Ini-error 和 Infil-error下得到模式模拟值(初 始场和模式预报均有误差), 同化时采用与模拟同样

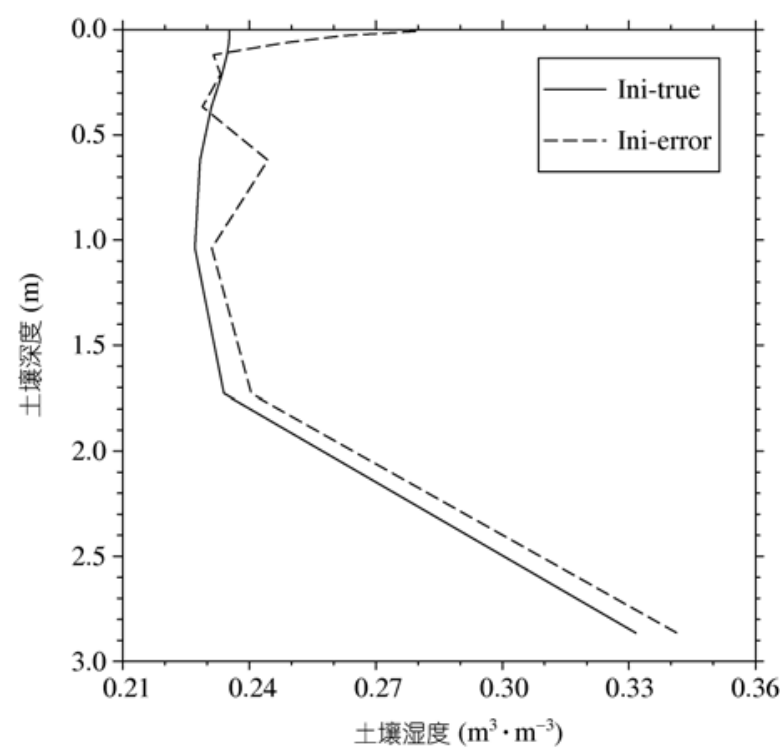

图 3 初始土壤湿度廓线分布 $\left(\theta_{0}\right)$

实线表示正确的初始场(Ini-true)，虚线表示有误差的初始场 (Ini-error)

的初始条件. 假设待优化的 3 个参数 $b^{\prime}, \sigma$ 和 $Q_{0}$ 选定 一组值作为真值, 然后用观测土壤湿度以及辅助数 据集驱动辐射传输模型得到观测亮温，这里假定辅 助数据集没有误差.

在理想试验中，对第一个阶段首先选取第 1 546 步进行参数优化, 其中 3 个待优化参数 $b^{\prime}, \sigma$ 和 $Q_{0}$ 取 一组任意值(有效范围内), 利用 SCE-UA 方法进行优 化. 第二个阶段在整个时间区间进行土壤湿度同化, 取初始场和预报模型都有误差，参数 $b^{\prime}, \sigma$ 和 $Q_{0}$ 取上一 阶段优化后平均值. 我们分别取 3.5, 7, $14 \mathrm{~h}$ 三种观测 频率进行同化试验，试验集合样本的个数取为 60 个. 
为了便于分析同化和模式模拟的效果, 定义如 下两个统计特征: 相对误差(relative error, RE)和均方 根误差(root mean square error, RMSE):

$$
\begin{gathered}
\mathrm{RE}=\frac{\left|\mathbf{S}_{i}-\mathbf{O}_{i}\right|}{\mathbf{O}_{i}}, \\
\mathrm{RMSE}=\sqrt{\frac{1}{n} \sum_{i=1}^{n}\left(\mathbf{S}_{i}-\mathbf{O}_{i}\right)^{2},}
\end{gathered}
$$

其中, $\mathbf{S}$ 和 $\mathbf{O}$ 分别表示 $n$ 维的模式模拟(或同化)和观 测的向量.
由于此理想试验中观测亮温是利用观测土壤湿 度“生成”，因此同化亮温后的土壤湿度精度“最多”只 能与同化观测土壤湿度的相同. 图 4 表示每 $14 \mathrm{~h}$ 引入 微波亮温观测, 土壤湿度同化、模式模拟和真值的比 较(仅显示第 $1,3,5,7$ 层). 从图 4(a) 可以发现: 本研 究所使用的双通同化方案(简写为 Ass-Tb)由于考虑 了参数优化过程，使得表层土壤湿度的同化结果不 仅明显比模式模拟更接近真值，而且非常接近同化 观测土壤湿度(简写为 Ass-SM)的结果. Ass-Tb 方案得 到的表层 $(0 \sim 0.0175 \mathrm{~m})$ 土壤湿度同化结果与观测
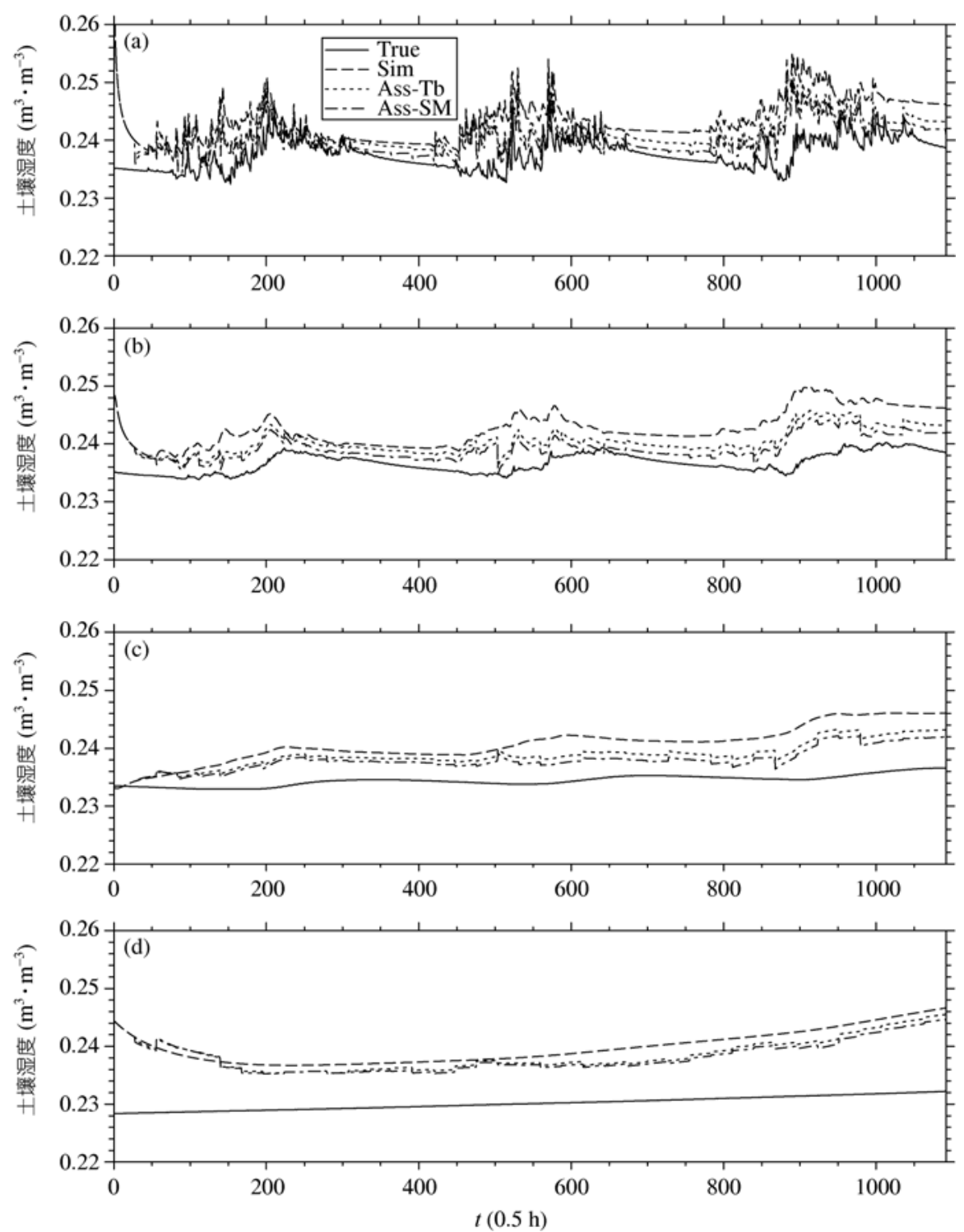

图 4 每 $14 \mathrm{~h}$ 引入观测, 同化、模拟(Sim)和真值(True)的比较

(a) (d)分别表示第 1(0 0.0175 m), 3(0.045 0.091 m), 5(0.17 0.29 m), 7(0.49 0.83 m)层; Ass-Tb 表示同化亮温, Ass-SM 表示同化观测土壤湿度 


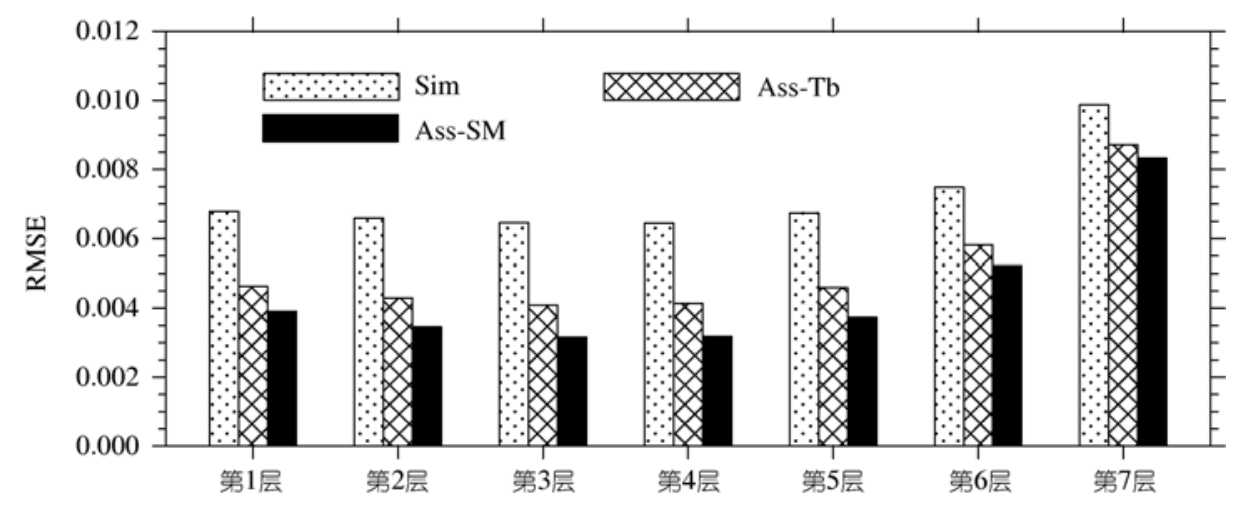

图 5 同化和模式模拟与真值时间序列的均方根误差(RMSE)

Sim 表示模拟, Ass-Tb 表示同化亮温, Ass-SM 表示同化观测土壤湿度

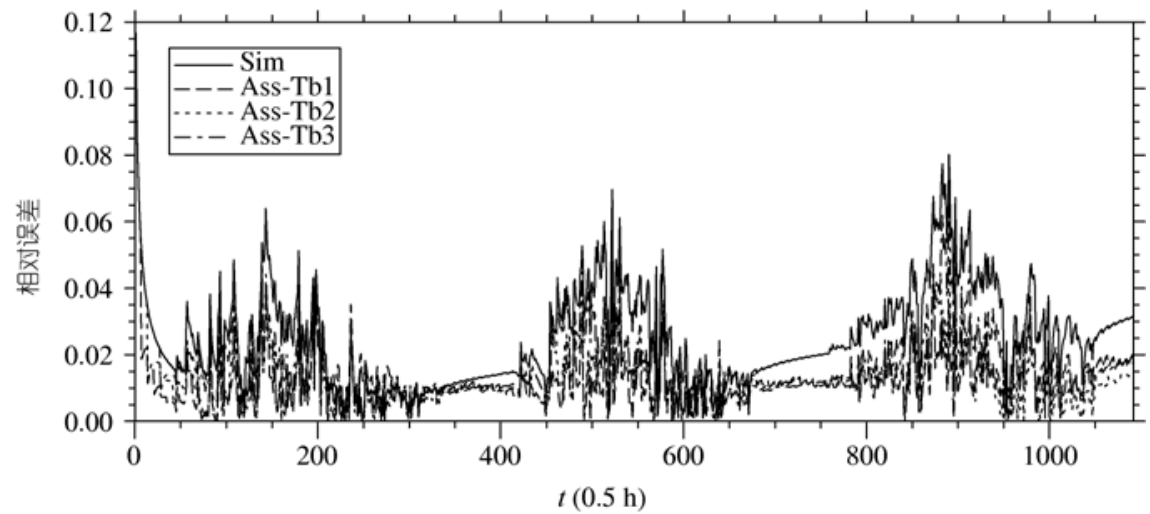

图 6 不同观测频率下同化和模式模拟的表层土壤湿度与真值的相对误差比较

$\operatorname{Sim}$ 为模式模拟, Ass-Tb1 表示 $3.5 \mathrm{~h}$ 引入一次观测亮温, Ass-Tb2 为 $7 \mathrm{~h} /$ 次, Ass-Tb3 为 $14 \mathrm{~h} /$ 次

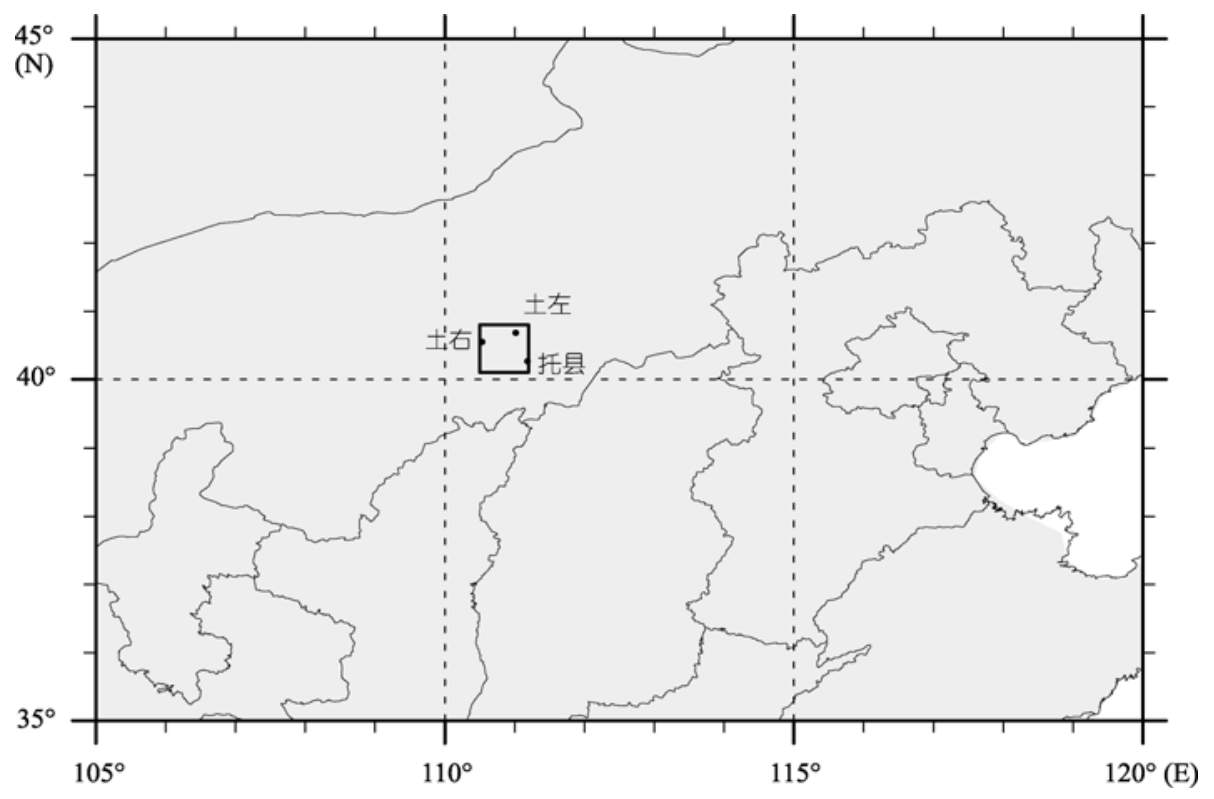

图 7 同化试验的研究区域 中心点 $\left(110.85^{\circ} \mathrm{E}, 40.85^{\circ} \mathrm{N}\right)$, 其 中托县 $\left(111.183^{\circ} \mathrm{E}, 40.267^{\circ} \mathrm{N}\right)$, 土左 $\left(110.533^{\circ} \mathrm{E}, 40.55^{\circ} \mathrm{N}\right)$, 土右 $\left(111.015^{\circ} \mathrm{E}, 40.683^{\circ} \mathrm{N}\right)$ 
序列的均方根误差(RMSE) 为 0.0356 , 而模式结果与 观测的 RMSE 为 0.0679 (图 5), 相对减小了 $47.6 \%$. Ass- $\mathrm{Tb}$ 方案对深层土壤湿度的模拟也有所改善(图 4(b) (d)), 相对于模式模拟结果, 同化结果和真值时 间序列的 RMSE 在第 3, 5, 7 层分别减小了 $36.6 \%$, $31.8 \%$ 和 11.7\%(图 5). 这主要是因为模式低估了表层 土壤湿度的模拟，尽管微波亮温仅含有表层土壤湿 度“信息”(0 1.75 cm), 但是通过同化亮温使得表层土 壤湿度的模拟变大更接近真值, 然后通过模式的动 力传输以及协相关可以将此 “信息”传递至较深层
$(1.75 \sim 83 \mathrm{~cm})$, 从而使得较深层土壤湿度得到一定程 度地改善 ${ }^{[11,20]}$. 但是更深层(超过 $1 \mathrm{~m}$ )土壤湿度由于 比较稳定，同化前后差别不大 ${ }^{[20]}$.

为了给出观测频率对同化结果的影响，下面设 计 1 组试验来验证它的敏感性(如图 6 所示): 每 3.5,7 和 $14 \mathrm{~h}$ 分别引入微波亮温观测. 图 6 表明, 随着观测 频率的减小, Ass- Tb 方案与真值的相对误差会有所增 大，但都优于模式模拟结果. 当每天一次亮温观测 (图中未显示), 同化结果比模式模拟的土壤湿度仍旧 有明显改善，这对于进行实测微波亮温(TMI, SSM/I,
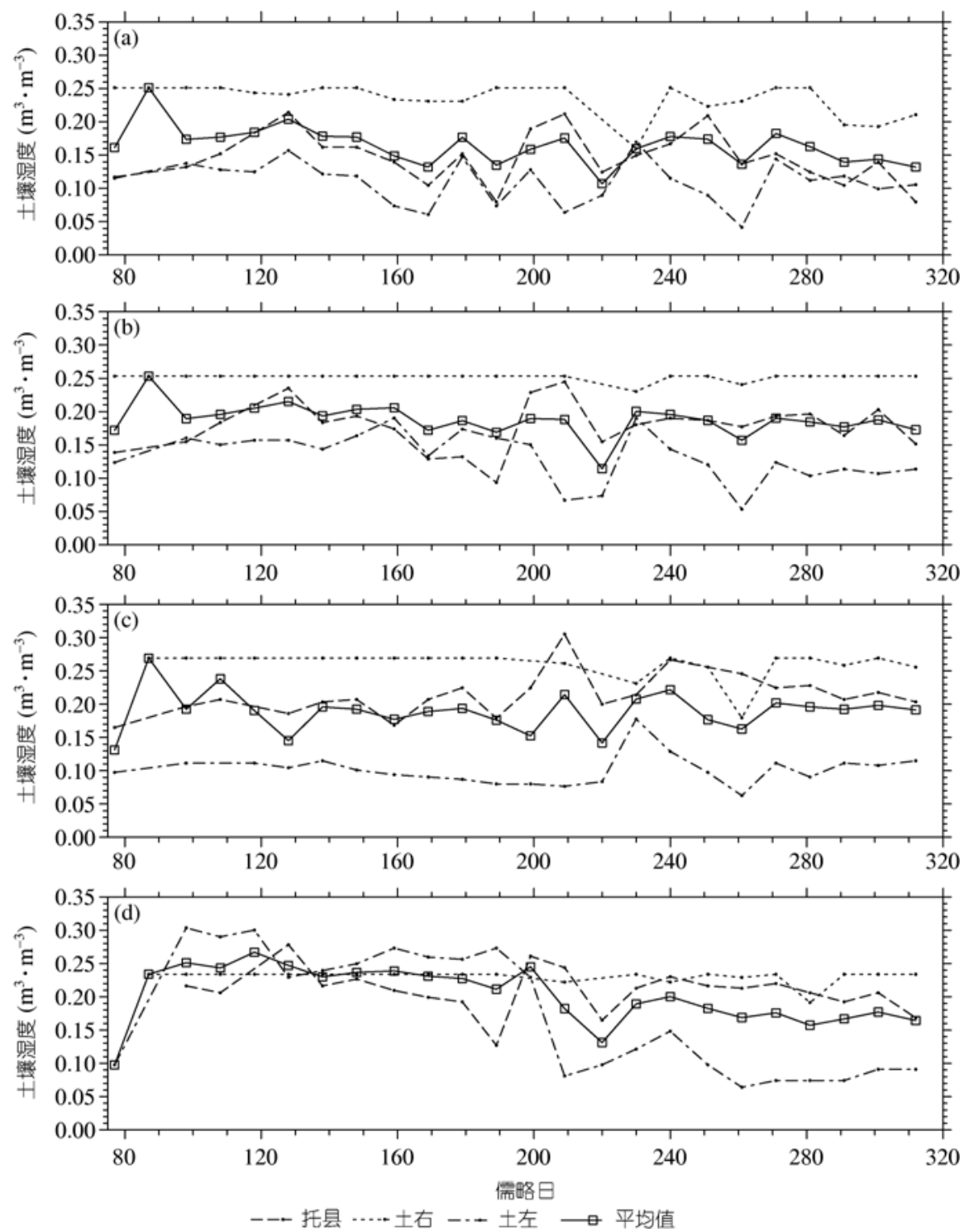

图 8 研究区域内 3 个站点在 2006 年 3 月 16 日 11 月 16 日期间的观测土壤湿度及其平均值

(a) 顶层 0 10 cm; (b) 第 2 层 10 20 cm; (c) 第 3 层 20 30 cm; (d) 第 4 层 40 50 cm 
AMSR-E 等)同化试验有很大的帮助, 下面将在内蒙 古地区对实测 AMSR-E 亮温资料进行同化试验.

\section{2 基于AMSR-E亮温的同化试验}

如图 7 所示, 选取位于内蒙古中部地区的一个 $0.7^{\circ} \times 0.7^{\circ}$ 网格, 中心点是 $\left(110.85^{\circ} \mathrm{E}, 40.85^{\circ} \mathrm{N}\right)$. 该地 区属于半干旱气候, 以草地为主, 下垫面比较均匀, 人类活动的影响较小. 在研究区域内有 3 个土壤湿度 观测站：托县、土右和土左, 每个月在 8 号、18 号和 28 号共有 3 次观测, 分布在 $0 \sim 10,10 \sim 20,20 \sim 30$ 和 40 50 cm 处. 本文选取 2006 年 3 月 8 日 11 月 8 日 期间的观测, 为了减小单点观测和网格尺度的不匹 配，用 3 个站点土壤湿度的平均值作为网格的“真实 观测值”来验证(图 8).

首先用 1973 2004 年大气强迫数据集 ${ }^{[40,41]}$ 驱动 CLM3.0 获得模式的平衡态, 然后在此基础上利用师
春香 ${ }^{[42]}$ 发展的高分辨率数据集 $\left(0.2^{\circ} \times 0.2^{\circ}\right)$ 通过 $\mathrm{CLM}$ 3.0 获得研究区域 2006 年 3 月 16 日 11 月 16 日的入 渗时间序列(图 9(a)) 以及辐射传输模型所需的辅助数 据集，如植被温度、地表温度、土壤质地和叶面积指 数等.

本文中 AMSR-E 亮温观测资料从美国科罗拉多 大学 ftp://sidads.colorado.edu 网站获得, 原始格式为 Ease-Grid(等面积可扩充地球网格). 选用 2006 年 3 月 16 日 11 月 16 日期间 $10.65 \mathrm{GHz}$ 亮温(垂直极化), 为 减小白天时各种干扰，试验中仅使用降轨资料(凌晨 过境), 并利用 http://nsidc.org 网站提供的相关说明和 程序将该亮温观测资料转化为经纬网投影(空间分 辨率约为 $25 \mathrm{~km})$, 对应文中研究区域的亮温见图 10(a).

由于本文发展的双通土壤湿度同化方案中辐射 传输模型并未考虑积雪覆盖的情形，因此只在 3 月
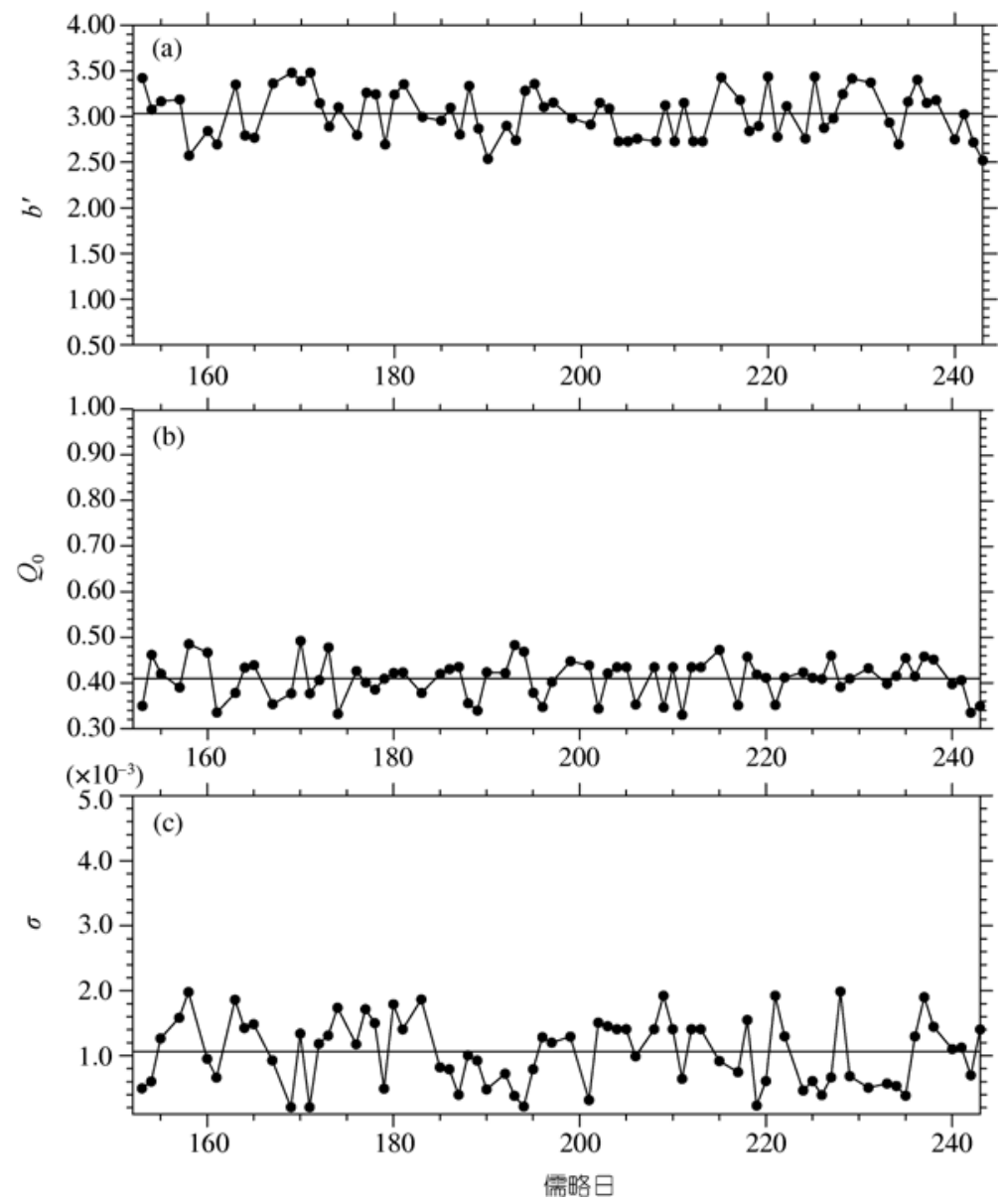

图 9 参数 $b^{\prime}, Q_{0}$ 和 $\sigma$ 在优化阶段 (2006 年 6 8 月)每天的优化结果(实心点) 水平直线表示各参数的平均值 

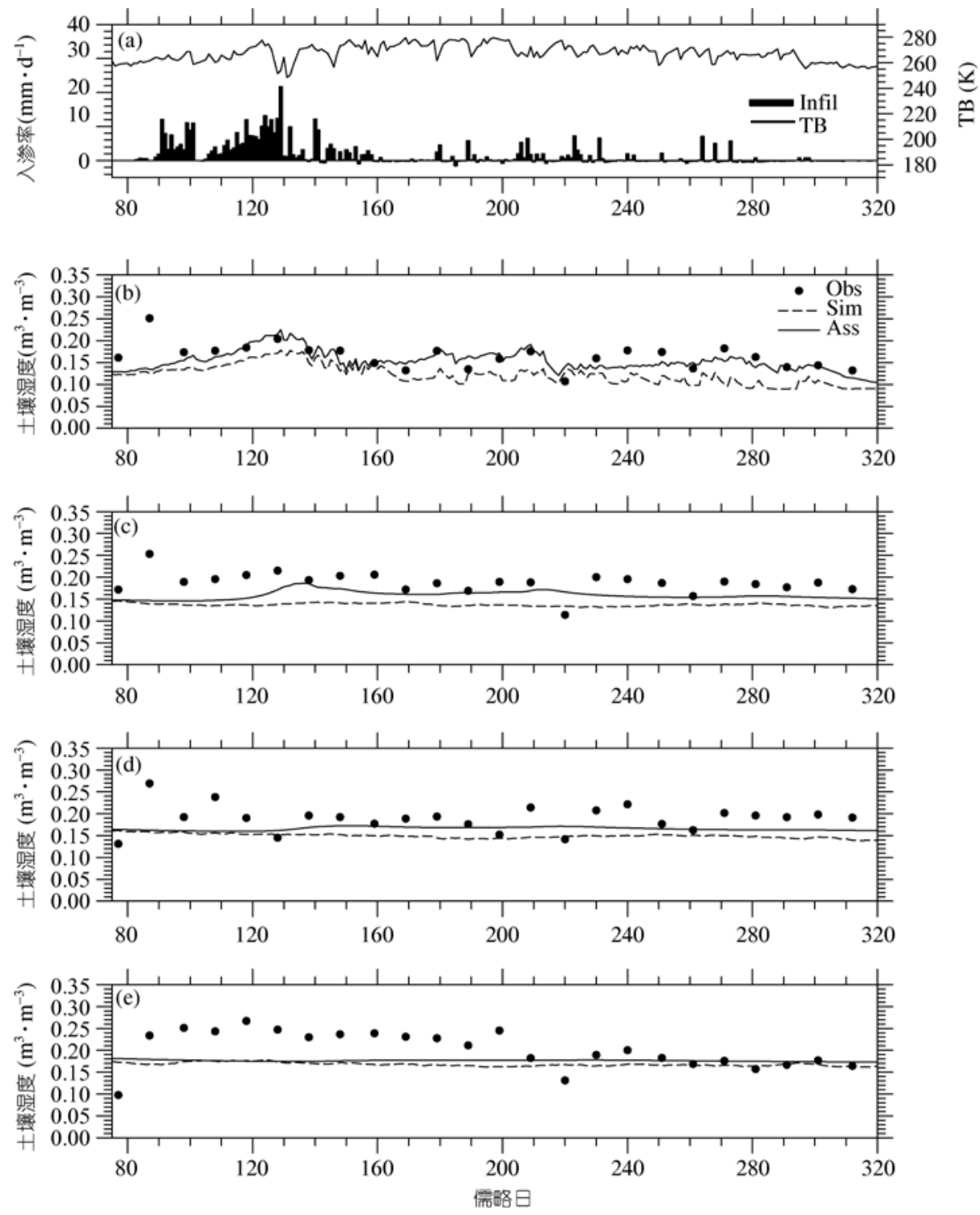

图 102006 年 3 月 16 日 11 月 16 日期间的入渗和 AMSR-E10.65 GHz 垂直极化亮温(a)及日平均土壤湿度时间序列 ((b) (e)) (b) 顶层 $(0 \sim 10 \mathrm{~cm})$; (c) 第 2 层 $(10 \sim 20 \mathrm{~cm})$; (d) 第 3 层 $(20 \sim 30 \mathrm{~cm})$; (e) 第 4 层 $(40 \sim 50 \mathrm{~cm})$. 其中, Infil 表示入渗, TB 表示亮温, Ass 为同化结果, Sim 表示模拟, Obs 为观测

16日 11月 16 日期间进行同化试验(儒略日第70 320 天). 本研究中所获得的土壤湿度台站观测资料的分层 与和陆面模式 CLM 3.0 的不同, 为了便于比较, 根据 CLM 3.0 的土壤分层把模式结果(包括同化和模拟)线 性插值到 0 10, 10 20, 20 30 和 40 50 cm 处.

首先利用 2006 年 6 8 月(儒略日第 152 243 天)3 个月作为参数优化阶段优化辐射传输模型的 3 个参 数 $b^{\prime}, \sigma$ 和 $Q_{0}$, 由于每天最多一次亮温观测, 我们选 取优化窗口长度为 $1 \mathrm{~d}$, 这些参数在优化阶段每天的
参数值以及平均值(实线)如图 9 所示. 由于 $b^{\prime}, \sigma$ 和 $Q_{0}$ 这 3 个参数从物理上属于静态参数, 但是由于大气驱 动数据以及辅助数据集等存在误差, 它们很难固定 不变. 根据图 9 可以发现, 这 3 个参数每天的值比较 分散, 但是均在某个范围内波动, 因此我们选取其平 均值作为最终优化参数值具有一定的合理性, 这是我 们对优化值求平均的主要原因. 由于这 3 个参数对辐 射传输模型的亮温模拟具有很大影响, 如果它们的分 散度很大, 而且很难确定时, 对于同化结果影响很大. 
各层土壤湿度的同化、模式模拟和观测的比较见 图 10. 图 10(b)表明同化 AMSR-E 亮温可以明显改善 顶层 $(0 \sim 10 \mathrm{~cm})$ 土壤湿度的估计, 同化结果不仅在量级 上与观测数据更吻合, 而且能够反映出顶层土壤湿度 的时间变异性. 其中, 相比较而言模式直接模拟的土 壤湿度则偏差较大, 顶层土壤湿度同化结果与观测的 RMSE 为 0.03355 , 比模拟的 0.05052 减小了 $33.6 \%$.

与理想试验结果类似, 较深层 $(10 \sim 50 \mathrm{~cm})$ 土壤湿 度同化结果比模拟也有一定程度的改善(图 10(b) (d)), 第 2 4 层 $(10 \sim 50 \mathrm{~cm})$ 同化与观测的 RMSE 比模 拟平均相对减小了 $20.9 \%$, 特别是第 2 层 $(10 \sim 20 \mathrm{~cm}$, 图 10(b)), 同化与观测的 RMSE 为 0.0415094 , 而模拟 为 0.05717589 . 尽管如此, 在较深层同化亮温有时候 可能会使模拟变得更糟糕, 例如儒略日第 230 天, 由 于模拟的土壤湿度在表层和深层均偏湿, 而表层的 同化结果比模拟还大，这种“信息”的往下传递使较深 层的土壤湿度比模拟还湿, 从而与观测偏离更远. 另 外, 当超过 $50 \mathrm{~cm}$ 时, 同化结果与土壤水模型模拟几 乎相同 (图中未显示), 改善并不明显. 这主要是因为 深区的土壤性质比较稳定, 使得土壤湿度变化幅度 不大而且深层土壤湿度受表层影响也很小 ${ }^{[43]}$, 这些 结论与 Zhang 等 ${ }^{[11]}$ 和 Huang 等 ${ }^{[20]}$ 的结果是一致的.

\section{3 小结和讨论}

本文以陆面过程模型 CLM 3.0 作为平台, 利用
其土壤水模型作为预报算子, 微波辐射传输模型作 为观测算子, 建立了基于集合 Kalman 滤波算法同化 微波亮温改善土壤湿度估计的双通同化方案. 由于 SCE-UA 优化算法的引入, 减少了辐射传输模型中植 被光学厚度和地表粗粘度参数的不确定性, 从而使 得微波遥感资料能更好地应用于陆面数据同化. 此 同化方案实现了辐射传输模型及顺序同化算法与陆 面过程模型 CLM 3.0 的“离线”耦合.

人工理想试验以及基于 AMSR-E 亮温的同化试 验都表明: 同化微波亮温能够明显改善表层土壤湿 度的模拟, 对较深层土壤湿度模拟也有一定程度的 改善, 而深层(超过 $50 \mathrm{~cm}$ ) 土壤湿度同化结果和模拟 差别不大, 主要是因为深层土壤性质比较稳定. 本研 究建立的双通同化方案对于土壤湿度观测稀少甚至 没有的地方尤其具有重要实际意义. 它可以利用同 化遥感资料来改善模式模拟，并且由于 AMSR-E 亮 温比土壤湿度站点观测具有更高的时空分辨率(约 25 $\mathrm{km}$ ), 此同化方案在获得更高精度的高时空分辨率土 壤湿度分布方面有较大的潜力。

需要指出的是, 文中辐射传输模型所需要的地 表温度、植被温度等是陆面过程模型 CLM 3.0 的输 出结果, 它们本身与实际值存在着偏差, 因此需要更 多的试验来确定它们对于土壤湿度同化结果的影响. 我们下一步将在此同化方案中考虑土壤冻融过程, 并完全耦合到陆面过程模型 CLM 3.0 中.

阳坤研究员在辐射传输模型方面提供帮助, 陈锋在画图方面提供帮助, 审稿人对本文提出建设性修改意见, 在此一并致谢.

\section{参考文献}

1 Entekhabi D, Rodriguez-Iturbe I, Castelli F. Mutual interaction of soil moisture state and atmospheric processes. J Hydrol, 1996, 184: 3-17

2 郭维栋, 马柱国, 王会军. 土壤湿度一一个跨季度降水预测中的重要因子及其应用探讨. 气候与环境研究, 2007, 12: 20一 28

3 Rodell M, Houser P R, Jambor U. The global land data assimilation system. Bull Amer Meteorol Soc, 2004, 85: 381-394

4 Entin J K, Robock A, Vinnikov K Y, et al. Evaluation of global soil wetness project soil moisture simulation. J Meteorol Soc Jpn, 1999, 77: $183-198$

5 Schmugge T J, O’Neill P E, Wang J R. Passive microwave soil moisture research. IEEE Trans Geosci Remote Sensing, 1986, 24: 12-22

6 Njoku E G, Jackson T J, Lakshmi V, et al. Soil moisture retrieval from AMSR-E. IEEE Trans Geosci Remote Sensing, 2003, 41: 215-229

7 Shi J C, Jiang L M, Zhang L X, et al. Physically based estimation of bare-surface soil moisture with the passive radiometers. IEEE Trans Geosci Remote Sensing, 2006, 44: 3145-3153

8 Reichle R H, Koster R D, Liu P, et al. Comparison and assimilation of global soil moisture retrievals from the Advanced Microwave Scanning Radiometer for the Earth Observing System (AMSR-E) and the Scanning Multichannel Microwave Radiometer (SMMR). J Geophys Res, 2007, 112: D09108, doi: 10.1029/2006JD008033 
9 Walker J P, Houser P R. A methodology for initializing soil moisture in a global climate model: Assimilation of near-surface soil moisture observations. J Geophys Res, 2001, 106: 11761-11774

10 Margulis S A, McLaughlin D, Entekhabi D, et al. Land data assimilation and estimation of soil moisture using measurements from the Southern Great Plains 1997 Field Experiment. Water Resour Res, 2002, 38: 1299, doi: 10.1029/2001WR001114

11 Zhang S W, Li H R, Zhang W D, et al. Estimating the soil moisture profile by assimilating near-surface observations with ensemble Kalman filter (EnKF). Adv Atmos Sci, 2006, 22: 936-945

12 黄春林, 李新. 土壤水分同化系统的敏感性试验研究. 水科学进展, 2006, 17: 457-465

13 张生雷, 谢正辉, 田向军, 等. 基于土壤水模型及站点资料的土壤湿度同化方法. 地球科学进展, 2006, 21: 1350-1362

14 张生雷, 谢正辉, 师春香, 等. 集合 Kalman 滤波在土壤湿度同化中的应用. 大气科学, 2008, 32: 1419一 1430

15 De Lannoy G J M, Houser P R, Pauwels V R N, et al. State and bias estimation for soil moisture profiles by an ensemble Kalman filter: Effect of assimilation depth and frequency. Water Resour Res, 2007, 43: W06401, doi: 10.1029/2006WR005100

16 Tian X J, Xie Z H, Dai A G. A land surface soil moisture data assimilation system based on the dual-UKF method and the Community Land Model. J Geophys Res, 2008, 113: D14127, doi: 10.1029/2007JD009650

17 田向军, 谢正辉. 考虑次网格变异性和土壤冻融过程的土壤湿度同化方案. 中国科学 D 辑: 地球科学, 2008, 38: 741一 749

18 Crosson W L, Laymon C A, Inguva R, et al. Assimilating remote sensing data in a Surface Flux-Soil Moisture Model. Hydrol Process, 2002, 16: $1645-1662$

19 Li X, Koike T, Mahadevan P. A very fast simulated re-annealing (VFSA) approach for land data assimilation. Comput Geosci, 2004, 30: $239-248$

20 Huang C L, Li X, Lu L, et al. Experiments of one-dimensional soil moisture assimilation system based on ensemble Kalman filter. Remote Sens Environ, 2008, 112: 888-900

21 Jakson T J, Schmugge T J. Vegetation effects on the microwave emission of soils. Remote Sens Environ, 1991, 36: 203-212

22 Wegmuller U, Matzler C. Rough bare soil reflectivity model. IEEE Trans Geosci Remote Sensing, 1999, 37: 1391-1395

23 Njoku E G, Chan S K. Vegetation and surface roughness effects on AMSR-E land observations. Remote Sens Environ, 2006, 100: 190199

24 Loew A. Impact of surface heterogeneity on surface soil moisture retrievals from passive microwave data at the regional scale: The upper Danube case. Remote Sens Environ, 2008, 112: 231-248

25 Yang K, Watanbe T, Koike T, et al. Auto-calibration system developed to assimilate AMSR-E data into a land surface model for estimating soil moisture energy budget. J Meteorol Soc Jpn, 2007, 85A: 229-242

26 Evensen G. Sampling strategies and square root analysis schemes for the EnKF. Ocean Dyn, 2004, 54: 539-560

27 Duan Q Y, Gupta V K, Sorooshian S. A shuffled complex evolution approach for effective and efficient global minimization. J Opt Theory App, 1993, 76: 501-521

28 Oleson K W, Dai Y J, Bonan G, et al. Technical description of the Community Land Model (CLM), NCAR/TN-461+STR, 2004

29 Fujii H. Development of a microwave radiative transfer model for vegetated land surface based on comprehensive in-situ observations. Doctoral Dissertation. Tokyo: University of Tokyo, 2005

30 Darcy H. The Public Fountains of the City of Dijon. Paris: Dalmount, 1856

31 谢正辉, 曾庆存, 戴永久, 等. 非饱和流问题的数值模拟研究. 中国科学 D 辑: 地球科学, 1998, 28: 175一-180

32 田向军. 包含地下水位动态变化的陆面水文机制集成及其与全球气候模式的双向耦合研究. 博士学位论文. 北京: 中国科学院研究 生院, 2006

33 Mo T, Choudury B J, Schmugge T J, et al. A model for microwave emission from vegetation covered fields. J Geophys Res, 1982, 87: $11229-11237$

34 Wang J R, Choudhury B J. Remote sensing of soil moisture content over bare fields at 1.4 GHz frequency. J Geophys Res, 1981, 86: 52775282

35 Dobson M C, Ulaby F T, Hallikainen M T, et al. Microwave dielectric behavior of wet soil-Part II: Dielectric mixing models. IEEE Trans Geosci Remote Sensing, 1985, 23: 35-46

36 Ulaby F T, Moore R K, Fung A K. Microwave Remote Sensing: Active and Passive-Volume III, From Theory to Applications. Norwood: Artech House, 1986

37 Paloscia S, Pampaloni P. Microwave polarization index for monitoring vegetation growth. IEEE Trans Geosci Remote Sensing, 1988, 26: $617-621$

38 Evensen G. The Ensemble Kalman Filter: Theoretical formulation and practical implementation. Ocean Dyn, 2003, 53: 343-367 
39 Duan Q Y, Sorooshian S, Gupta V K. Optimal use of the SCE-UA global optimization method for calibrating watershed models. J Hydrol, 1994, 158: 265-284

40 Qian T T, Dai A G, Trenberth K, et al. Simulation of global land surface conditions from 1948 to 2004, part I: Forcing data and evaluations. J Hydrometeorol, 2006, 7: 953-975

41 Tian X J, Dai A G, Yang D Q, et al. Effects of precipitation-bias corrections on surface hydrology over northern latitudes. J Geophys Res, 2007, 112: D14101, doi: 10.1029/2007JD008420

42 师春香. 基于 EnKF 算法的卫星遥感土壤湿度同化研究. 博士学位论文. 北京: 中国科学院研究生院, 2008

43 Yang K, Koike T. Inverse analysis of the role of soil vertical heterogeneity in controlling surface soil state and energy partition. J Geophys Res, 2005, 110: D08101, doi: 10.1029/2004JD005500 